\title{
Knowledge and Attitudes of Nurses Working at King Abdulaziz University Hospital toward Cancer Pain Management
}

\author{
Rolina K. Al-Wassia \\ Department of Radiology, King Abdulaziz University Hospital, Jeddah, Saudi Arabia \\ Email: ralwassia@kau.edu.sa
}

Received 16 March 2016; accepted 16 April 2016; published 19 April 2016

Copyright (C) 2016 by author and Scientific Research Publishing Inc.

This work is licensed under the Creative Commons Attribution International License (CC BY). http://creativecommons.org/licenses/by/4.0/

(c) (i) Open Access

\begin{abstract}
Objective: This survey aims to identify the levels of knowledge and attitudes among nurses regarding cancer pain management. Methods: This cross-sectional survey was undertaken at King Abdulaziz University Hospital, Jeddah, between September 4 and September 27, 2015. The survey instrument was a pre-set questionnaire comprising 39 closed-ended format questions. Participants were asked questions to assess their knowledge and attitudes about cancer pain management and adherence to frequent misconceptions regarding opioid therapy. The chi-square test was used to compare differences between variables. Results: One hundred twenty-eight questionnaires were completed and analyzed. A mean sample score of $41.3 \%$ was achieved on pain-related knowledge questions. The average score on all 39 questions was $16.1 \pm 4.6$ (range, 0 - 24). Nurses on the male medical ward were most knowledgeable compared with those on other wards $(p<$ 0.001). They were also most likely to score highest on questions that assessed knowledge of pain management $(p<0.001)$. Conversely, nurses on the female surgical ward were most likely to score highest on issues related to pain assessment $(p<0.001)$ and experience in managing breakthrough cancer pain $(p<0.001)$. Likewise, nurses on the male surgical and female medical wards appeared to be more knowledgeable on issues related to patient compliance $(p=0.002)$. Conclusion: Optimization of inpatient supportive procedures should be a specific task at King Abdulaziz University Hospital until an oncology unit with nurses specialized in cancer care is established.
\end{abstract}

\section{Keywords}

Attitude, Breakthrough Cancer Pain, Cancer, Knowledge, Nurse, Pain Management

\section{Introduction}

Over the last decade, the number of cancer patients diagnosed and treated at King Abdulaziz University Hospital 
(KAUH) has increased steadily [1]-[6]. Cancer patients are frequently admitted to our hospital due to acute conditions, including pain, shortness of breath, bleeding, and headache or other refractory symptoms related to the tumor location. The emergency department admits these patients to the related medical, surgical, and gynecological wards since the hospital lacks a dedicated oncology unit with specialized oncology nurses to manage cancer cases.

Cancer patients develop many health and psychological issues that have to be addressed by a specialized team. Unfortunately, cancer pain is still considered one of the most feared consequences in cancer patients. It has a profound impact on every aspect of quality of life and, therefore, is considered the leading cause of suffering in cancer patients [7] [8].

Persistent pain is also a cause of distress for the patient's relatives, who usually feel that the medical staff should be able to control the pain even if the cancer itself cannot be controlled. When the pain is not controlled, relatives are frustrated, and their resentment is usually a source of job dissatisfaction for the medical staff [9].

In a meta-analysis conducted in 2007 by the European Society of Medical Oncology [10], it was shown that the pooled prevalence of cancer pain was $>50 \%$ in all cancer types, with the highest prevalence in head/neck cancer patients (70\%). In a recent systematic study that reviewed the barriers hindering adequate cancer pain management, Oldenmenger [11] showed that the most frequently mentioned barriers for both patients and professionals were knowledge deficits, inadequate pain assessment, and misconceptions regarding pain.

In spite of the fact that pain relief is achievable in more than $90 \%$ of cancer cases according to the World Health Organization's Cancer Pain Management guidelines [12] [13], inadequate pain relief in practice is well documented worldwide [14] [15]. The lack of knowledge regarding pain management by healthcare professionals - both practicing physicians and nurses - has emerged as one of the most significant problems related to effective pain management worldwide. This lack of knowledge can hinder the trust of cancer patients expecting a high level of communication and pain symptom control from their healthcare providers [16].

The purpose of this groundbreaking survey at KAUH was to identify the level of knowledge and attitudes among nurses regarding the management of cancer pain.

\section{Materials and Methods}

\subsection{Design and Participants}

This cross-sectional survey was undertaken at KAUH, Jeddah, Saudi Arabia, between September 4 and September 27, 2015 (A total of 207 nurses working at the emergency, male medical, female medical, male surgical, female surgical, or gynecology wards of KAUH were invited to participate in this survey). Of these, 128 filled in and returned the questionnaires, representing an overall response rate of $61 \%$. With regard to sample size the subjects included in the study are all the nurses working in the male surgical, female surgical, male medical, female medical, Gynecology wards and emergency department. All nurses at the pediatric and obstetric wards were excluded from this study, because nurses working in these wards do not care of cancer patients. Informed consent was sought from all participants prior to recruitment. The Research Ethics Committee at King Abdulaziz University approved this study.

\subsection{Survey Instrument}

The "Knowledge and Attitudes Survey Regarding Pain" tool developed by Ferrell and Bennet (B. \& M., 2014) was administered in this study. The instrument was developed in 1978 and has been developed over the course of time. The content of the tool was derived from current standards of pain management as defined by the World Health Organization, the American Pain Society, and the National Comprehensive Cancer Network Pain Guidelines. Construct validity was established by comparing nurses' scores at different levels of expertise, including students, fresh and former graduates, oncology nurses and senior pain experts. For the English version of the instrument, test-retest reliability was $>0.80$ and the alpha was $>0.7$.

The survey instrument was a pre-set questionnaire comprising 39 closed-ended format questions. The wording of survey questions was refined through a pilot study. Participants were asked questions to assess their knowledge and attitudes about cancer pain management and adherence to frequent misconceptions regarding opioid therapy reported in previous studies [10] [16] [17]. The items were selected to indicate misconceptions that may preclude optimal pain management in our setting. Topics for these items were divided into four main categories: pain management (questions 5 - 12,15, 17, 19 - 22, 24 - 26, 29, 36, and 37); pain assessment (ques- 
tions 1 - 4, 13, 16, 18, 30 - 32, 36, and 37); and nurses' experience in characterizing and treating breakthrough cancer pain (questions 23, 28, 33, 34, 36, and 37); and patient compliance (questions 14, 27, and 35). Each correct answer was given a score of 1 and each wrong one a score of 0 . Mean scores were compared between nurses working at all the surveyed wards. Items that had desired responses of $\geq 80 \%$ were regarded satisfactory and interpreted to indicate small to no substantial knowledge gaps, whereas those that achieved desired response rates $<50 \%$ were considered unsatisfactory.

\subsection{Data Analysis}

This study was analyzed using the Statistical Package for the Social Sciences (IBM, New York, US), version 22. Descriptive statistics were used to define the characteristics of the study variables through counts and percentages for categorical and nominal variables while continuous variables are presented as means and standard deviations. The chi-square test was used to compare differences between variables. Differences were considered statistically significant at a p-value of $<0.05$.

\section{Results}

A total of 128 questionnaires were completed and analyzed: 38 from nurses on the emergency ward, 30 from the male medical ward, 29 from the female medical ward, 24 from the male surgical ward, 5 from the female surgical ward, and 7 from the gynecology ward. The respective response rates were as follows: emergency ward, $44.0 \%$; male medical ward, $61.2 \%$; female medical ward, 59.2\%; male surgical ward, $60.0 \%$; female surgical ward, $18.5 \%$, and gynecology ward, $58.3 \%$.

In general, performance on the pain-related knowledge questions was slightly below average, with a mean sample score of $41.3 \%$. The average score on all 39 questions was $16.1 \pm 4.6$ (range, 0 - 24). A comparison of mean scores revealed that highest scores were achieved on issues related to nursing management of pain in cancer patients (mean, $9.8 \pm 2.6$ ). Conversely, patient compliance issues attained the lowest scores (mean, $0.9 \pm 0.6$; Table 1). Furthermore, nurses had insufficient experience in characterizing and treating breakthrough cancer pain.

A breakdown of correct responses to all 39 questions is shown on Table 2. Nurses performed best on questions $7,15,21,23,26$ and 30, with 91.1\% $(\mathrm{n}=112)$ responding correctly to question 21 (Figure 1$)$. They performed poorly in 23 of the questions, with the poorest responses $(n=1 ; 0.9 \%)$ documented for question 36B (Figure 2).

Further analysis revealed that nurses on the male medical ward were most knowledgeable compared with those on all other surveyed wards ( $p<0.001$; Table 3 ). They were also most likely to score highest on questions that assessed pain management $(p<0.001)$. Nurses on the female surgical ward were most likely to score highest on questions that concerned pain assessment and experience in characterizing and treating breakthrough cancer pain $(p<0.001)$. Nurses on the male surgical and female medical wards appeared to be more knowledgeable on issues related to patient compliance $(p=0.002)$.

\section{Discussion}

The way nurses perceive pain in cancer patients is important for the optimal management of pain; therefore, knowledge deficits and improper attitudes may hinder their appropriate responses to patients' pain relief needs.

Table 1. Comparison of mean scores of different knowledge questions by category.

\begin{tabular}{ccc}
\hline Variables & Range & Mean (SD) \\
\hline Pain management & $0-15$ & $9.8(2.6)$ \\
Pain assessment & $0-10$ & $4.6(2.1)$ \\
Nurses experience in characterizing and treating BTCP & $0-6$ & $3.6(1.5)$ \\
Patient compliance & $0-2$ & $0.9(0.6)$ \\
\hline
\end{tabular}

Abbreviations: BTCP, breakthrough cancer pain; SD, standard deviation. 
Table 2. Proportion of correct responses by the nurses.

\section{Questions}

Frequency (percent)

1. Vital signs are always reliable indicators of the intensity of a patient's pain.

$19(15.2 \%)$

2. Because their nervous system is underdeveloped, children under two years of age have decreased pain sensitivity and limited memory of painful experiences.

$44(36.7 \%)$

3. Patients who can be distracted from pain usually do not have severe pain.

4. Patients may sleep in spite of severe pain.

5. Aspirin and other nonsteroidal anti-inflammatory agents are NOT effective analgesics for painful bone metastases.

6. Respiratory depression rarely occurs in patients who have been receiving stable doses of opioids over a period of months.

7. Combining analgesics that work by different mechanisms (e.g., combining an NSAID with an opioid) may result in better pain control with fewer side effects than using a single analgesic agent.

8. The usual duration of analgesia of 1-2 mg morphine IV is 4 - 5 hours.

9. Research shows that promethazine (Phenergan) and hydroxyzine (Vistaril) are reliable potentiators of opioid analgesics.

10. Opioids should not be used in patients with a history of substance abuse.

11. Elderly patients cannot tolerate opioids for pain relief.

12. Patients should be encouraged to endure as much pain as possible before using an opioid.

$40(32.8 \%)$

13. Children less than 11 years old cannot reliably report pain so clinicians should rely solely on the parent's assessment of the child's pain intensity.

14. Patients’ spiritual beliefs may lead them to think pain and suffering are necessary.

15. After an initial dose of opioid analgesic is given, subsequent doses should be adjusted in accordance with the individual patient's response.

16. Giving patients sterile water by injection (placebo) is a useful test to determine if the pain is real.

17. Vicodin (hydrocodone $5 \mathrm{mg}+$ acetaminophen $500 \mathrm{mg}$ )

PO is approximately equal to 5 - $10 \mathrm{mg}$ of morphine PO.

18. If the source of the patient's pain is unknown, opioids should not be used during

the pain evaluation period, as this could mask the ability to correctly diagnose the cause of pain.

19. Anticonvulsant drugs such as gabapentin (Neurontin) produce optimal pain relief after a single dose.

20. Benzodiazepines are not effective pain relievers unless the pain is due to muscle spasm.

by behaviors that include one or more of the following: impaired control

over drug use, compulsive use, continued use despite harm, and craving.

22. The recommended route of administration of opioid analgesics

for patients with persistent cancer-related pain is:

23. The recommended route administration of opioid analgesics for patients with brief, severe pain of sudden onset such as trauma or postoperative pain is:

24. Which of the following analgesic medications is considered the drug of choice for the treatment of prolonged moderate to severe pain for cancer patients?

25. Which of the following intravenous doses of morphine administered over a 4 hour period would be equivalent to $30 \mathrm{mg}$ of oral morphine given 4 hours?

26. Analgesics for post-operative pain should initially be given

$10(8.4 \%)$

Yesterday the patient was receiving morphine $200 \mathrm{mg} /$ hour intravenously.

Today he has been receiving $250 \mathrm{mg}$ /hour intravenously. The likelihood of the patient developing clinically 


\section{Continued}

28. The most likely reason a patient with pain would request increased doses of pain medication is:

$86(72.3 \%)$

29. Which of the following is useful for treatment of cancer pain?

$60(50.0 \%)$

30. The most accurate judge of the intensity of the patient's pain is

31. Which of the following describes the best approach for cultural considerations in caring for patients in pain?

33. The time to peak effect for morphine given IV is

34. The time to peak effect for morphine given orally is

35. Following abrupt discontinuation of an opioid, physical dependence is manifested by the following:

36A. On the patient's record you must mark his pain on the scale below.

Circle the number that represents your assessment of Andrew’s pain.

$63(55.8 \%)$

36B. Your assessment, above, is made two hours after he received morphine $2 \mathrm{mg}$ IV.

Half hourly pain ratings following the injection ranged from 6 to 8 and he had

no clinically significant respiratory depression, sedation, or other untoward side effects.

37A. On the patient's record you must mark his pain on the scale below.

Circle the number that represents your assessment of Robert's pain:

Your assessment, above, is made two hours after he received morphine $2 \mathrm{mg} I V$. Half hourly pain ratings following the injection ranged from 6 to 8 and he had no clinically significant respiratory depression, sedation, or other untoward side effects

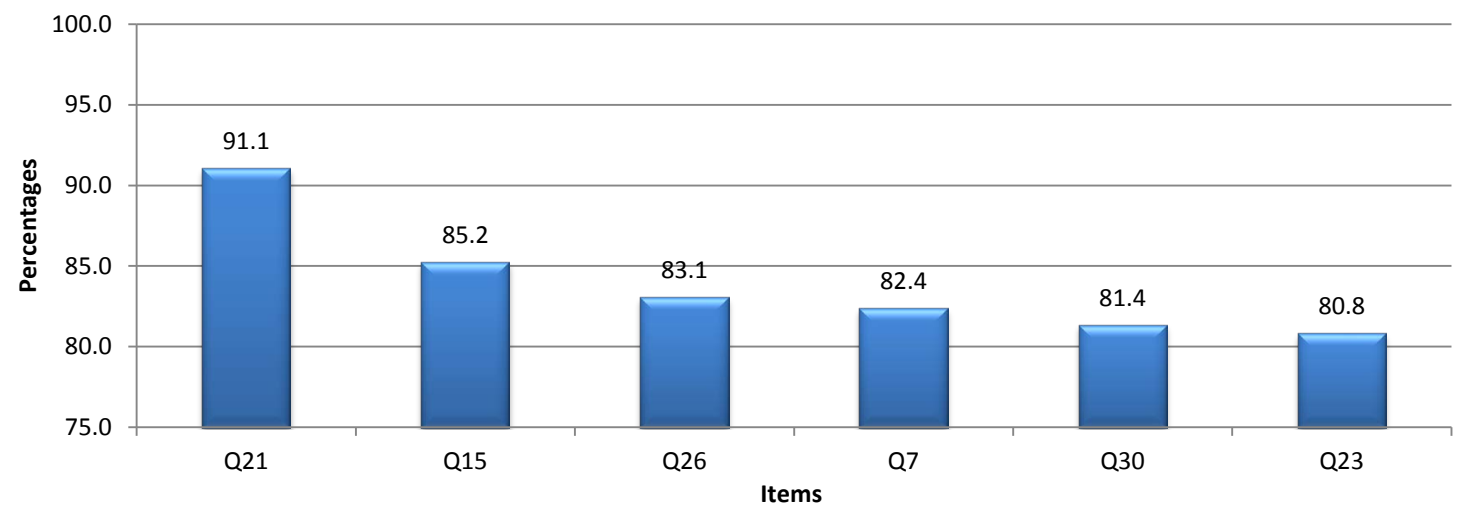

Figure 1. Knowledge and attitude items that had desired responses of $80 \%$ or more of the respondents.

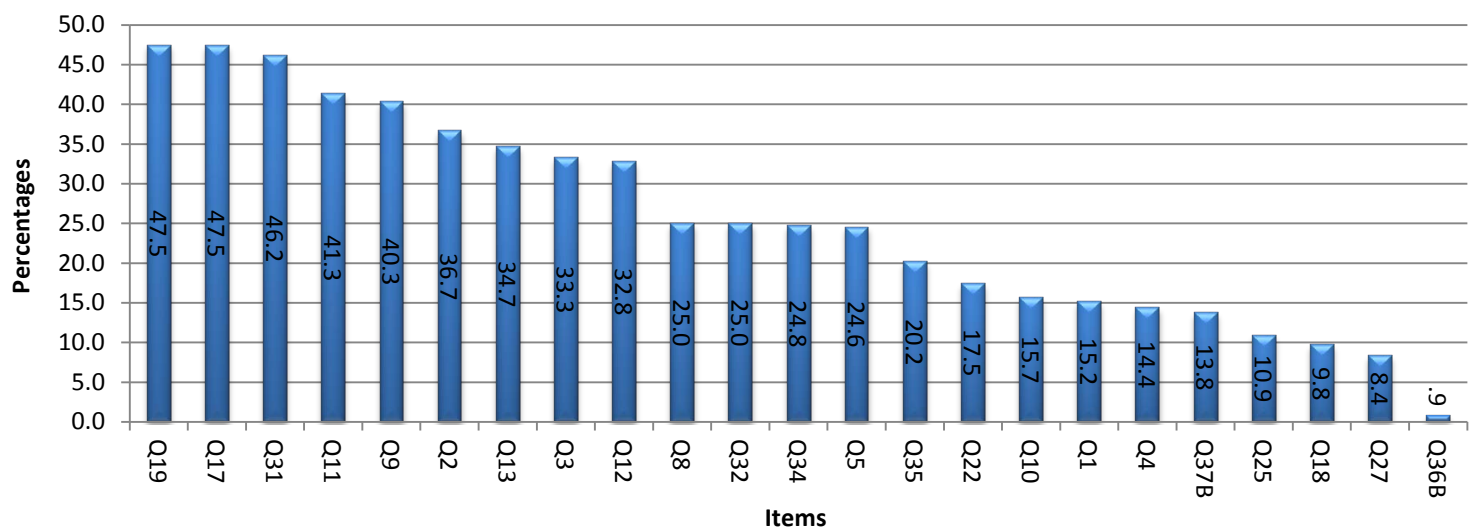

Figure 2. Knowledge and attitude items that had responses of $50 \%$ or less of the respondents. 
Table 3. Comparison of nurses mean scores on knowledge and attitude questions ${ }^{\mathrm{a}}$.

\begin{tabular}{cccccccc}
\hline Variables & $\begin{array}{c}\text { Male } \\
\text { surgical ward }\end{array}$ & $\begin{array}{c}\text { Female } \\
\text { surgical } \\
\text { ward }\end{array}$ & $\begin{array}{c}\text { Female } \\
\text { medical } \\
\text { ward }\end{array}$ & $\begin{array}{c}\text { Gynecology } \\
\text { ward }\end{array}$ & $\begin{array}{c}\text { Male } \\
\text { medical } \\
\text { ward }\end{array}$ & ER & $\boldsymbol{p}$-value \\
\hline Pain knowledge & $14.7 \pm 5.6$ & $19.4 \pm 2.6$ & $15.5 \pm 3.7$ & $10.0 \pm 0.6$ & $19.8 \pm 2.2$ & $15.2 \pm 4.5$ & $<0.001^{\mathrm{b}}$ \\
Pain management & $9.2 \pm 3.2$ & $11.0 \pm 1.6$ & $9.0 \pm 2.4$ & $7.9 \pm 0.7$ & $11.5 \pm 1.6$ & $9.7 \pm 2.9$ & $<0.001^{\mathrm{b}}$ \\
$\begin{array}{c}\text { Pain assessment } \\
\text { Nurses experience in }\end{array}$ & $3.5 \pm 1.7$ & $7.0 \pm 1.9$ & $3.9 \pm 1.5$ & $0.9 \pm 0.7$ & $6.5 \pm 1.4$ & $4.7 \pm 1.8$ & $<0.001^{\mathrm{b}}$ \\
characterizing and treating BTCP & $2.4 \pm 1.6$ & $4.4 \pm 0.9$ & $3.8 \pm 1.4$ & $0.4 \pm 0.5$ & $4.3 \pm 0.6$ & $4.2 \pm 0.8$ & $<0.001^{\mathrm{b}}$ \\
Patient compliance & $1.1 \pm 0.3$ & $1.0 \pm 1.0$ & $1.1 \pm 0.6$ & $0.9 \pm 0.4$ & $0.8 \pm 0.6$ & $0.6 \pm 0.5$ & $0.002^{\mathrm{b}}$ \\
\hline
\end{tabular}

Abbreviations: BTCP, breakthrough cancer pain; ER, emergency room; SD, standard deviation. ${ }^{\text {a }}$ The data are presented as mean \pm SD unless otherwise specified. 'Significant using one-way ANOVA at $p<0.05$.

Overall, this sample of nurses appeared to have insufficient knowledge and inappropriate attitudes about cancer pain management, as determined by their overall scores. Furthermore, when individual items were analyzed, we identified specific knowledge gaps in pain assessment and management, which are similar to those reported about nurses in other countries [18]-[20].

It is important that nurses are unequivocal about their beliefs and attitudes regarding cancer pain management before attempting to address the concerns of their patients. Unfortunately, gaps in cancer pain management and inadequate attitudes are not uncommon among nurses. Similar to our report, other authors [18]-[20] found that nurses had knowledge deficits and inappropriate attitudes about cancer pain management. In one survey that sought to examine nurses' knowledge and attitudes related to pain management among a sample of Australian acute care nurses [21], it was found that performance on the formal measure of pain-related knowledge was of moderate standard. However, contrary to our report, where we found a mean sample score of $41.3 \%$ on the pain-related knowledge questions, those authors found a correct rate of about $61.0 \%$. Further, they found that pharmacological management was the domain that attained the least scores, with a correct rate of $51.0 \%$. While we did not categorize our survey questions into pharmacological management or treatment domains, an analysis of individual items showed that nurses performed poorly on issues related to pain management and pharmacological treatment of pain in cancer patients.

In this study, nurses had poor experience in managing cancer pain. Several factors may explain this observation: (a) fear of patient addiction to opioid medications, (b) concern about the adverse effects of the medications, and (c) fear of tolerance to the drug. This finding has also been reported by other authors who, in addition, found that nurses faced difficulties in differentiating breakthrough cancer pain from insufficiently controlled background pain [22]. It is plausible that the difficulties encountered by our nurses may be due to their inability to identify breakthrough cancer pain as a subtype that occurs in spite of relatively optimally controlled background pain. However, a number of factors are reportedly involved in the undertreatment and management of breakthrough cancer pain, which may explain the differences observed between nurses in different regions. These include cultural, educational, logistic, health resource utilization, political, and religious factors [23] as well as those related to patients and to the healthcare staff [15]. A point of concern is the fact that only $55.8 \%$ of our nurses could correctly assess the severity of a patient's pain and only $0.9 \%$ could correctly determine the right action to take after assessing a patient two hours following intravenous morphine (2 mg).

Most studies pertaining to nurses' knowledge and attitudes toward cancer pain management focused on specific nurse populations and practice settings [18] [19] [21] [22] [24]. In this study, however, we included nurses working on different wards at an academic teaching center. While there is no clear explanation why nurses on specific wards appeared to be more knowledgeable than those on other wards, the differences observed between the nurses may be due to differences in routines. In addition, it is plausible that nurses on particular wards have little or no access to Continuing Medical Education activities specific to the management of cancer pain. In a previous study that assessed knowledge and attitudes toward pain management between oncology and nononcology nurses [25]-[27], the authors found that oncology nurses understood pain management principles than their nononcology counterparts. It was suggested that knowledge differences between the two groups were probably related to the educational preparation of oncology nurses to pain management courses, which were additional educational programs or post registration training courses [28] [29]. As a result, most oncology nurses 
were better prepared to management pain and recognize the importance of individual pain management tasks, whether these were pharmacological or nonpharmacological. While some researchers [30] reported that nurses working on oncology wards were concerned about taking more courses on analgesia, other investigators [28] [29] [31] found that nurses were not confident about their knowledge of pain management since their basic training did adequately prepare them to care for cancer patients in pain.

Although this study is the first attempt to assess nurses’ knowledge and attitudes regarding cancer pain management at our institution, it should be interpreted in light of its limitations. The cross-sectional design of this survey and the lack of information about the influence of demographic variables preclude any inferences being drawn about the correlation between demographic characteristics and cancer pain knowledge. In addition, our study was based on a small, single-center sample of nurses at five wards of our institution. Thus, our findings cannot be extrapolated to other groups, such as nurses on the pediatric wards. Further research will be needed to investigate the influence of demographic variables on cancer pain management concept.

However, this survey is a preliminary step to help decision makers design workshops that address important areas in cancer pain management. A follow-up and second assessment will be necessary to evaluate the benefits gained from organizing such workshops and subsequently help the administration to create long-term programs that will help improve the care of cancer patients at our institution.

\section{Conclusion}

In conclusion, inpatient care is probably an unavoidable step in the cancer trajectory. Until the establishment of an oncology unit with nurses specialized in cancer care, optimization of inpatient supportive procedures should be a specific task at King Abdulaziz University Hospital. Alternatively, consideration should be given to develop training programs aimed at educating nurses on issues related to pain management, pain assessment, breakthrough cancer pain, and patient compliance.

\section{References}

[1] Al-Ahwal, M.S. (2006) HER-2 Positivity and Correlations with Other Histopathologic Features in Breast Cancer Patients-Hospital Based Study. Journal of Pakistan Medical Association, 56, 65-68.

[2] Sait, K.H. (2011) Early Experience with the da Vinci Surgical System Robot in Gynecological Surgery at King Abdulaziz University Hospital. International Journal of Women's Health, 3, 219-226. http://dx.doi.org/10.2147/IJWH.S23046

[3] Alabbas, F.F., Al-Otaibi, S.M., Pasha, M.H., Alghamdi, A.M., Al-Hindi, H.M., Al-Ahwal, M.S., et al. (2015) Impact of Physiological Symptoms and Complications of Colorectal Cancer on the Quality of Life of Patients at King Abdulaziz University Hospital. Journal of Cancer Education, 1-7. http://dx.doi.org/10.1007/s13187-015-0839-7

[4] Mosli, H.A. (1997) Prostate Cancer: Experience at King Abdulaziz University Hospital, Jeddah. Annals of Saudi Medicine, 17, 590-594.

[5] Rouzi, A.A., Sahly, N.N., Sahly, N.F. and Alahwal, M.S. (2009) Cisplatinum and Docetaxel for Ovarian Cancer in Pregnancy. Archives of Gynecology and Obstetrics, 280, 823-825. http://dx.doi.org/10.1007/s00404-009-0992-5

[6] Alamoudi, O.S. (2010) Lung Cancer at a University Hospital in Saudi Arabia: A Four-Year Prospective Study of Clinical, Pathological, Radiological, Bronchoscopic, and Biochemical Parameters. Annals of Thoracic Medicine, 5, 30-36. http://dx.doi.org/10.4103/1817-1737.58957

[7] Grond, S., Zech, D., Diefenbach, C. and Bischoff, A. (1994) Prevalence and Pattern of Symptoms in Patients with Cancer Pain: A Prospective Evaluation of 1635 Cancer Patients Referred to a Pain Clinic. Journal of Pain and Symptom Management, 9, 372-382. http://dx.doi.org/10.1016/0885-3924(94)90174-0

[8] Chapman, C.R. and Gavrin, J. (1999) Suffering: The Contributions of Persistent Pain. The Lancet, 353, $2233-2237$. http://dx.doi.org/10.1016/S0140-6736(99)01308-2

[9] LeBaron, V., Beck, S.L., Black, F. and Palat, G. (2014) Nurse Moral Distress and Cancer Pain Management: An Ethnography of Oncology Nurses in India. Cancer Nursing, 37, 331-344. http://dx.doi.org/10.1097/ncc.0000000000000136

[10] van den Beuken-van Everdingen, M.H, de Rijke, J.M., Kessels, A.G., Schouten, H.C., van Kleef, M. and Patijn, J. (2007) Prevalence of Pain in Patients with Cancer: A Systematic Review of the Past 40 Years. Annals of Oncology, 18, 1437-1449. http://dx.doi.org/10.1093/annonc/mdm056

[11] Oldenmenger, W.H., Sillevis Smitt, P.A., van Dooren, S., Stoter, G. and van der Rijt, C.C. (2009) A Systematic Review on Barriers Hindering Adequate Cancer Pain Management and Interventions to Reduce Them: A Critical Ap- 
praisal. European Journal of Cancer, 45, 1370-1380. http://dx.doi.org/10.1016/j.ejca.2009.01.007

[12] Schug, S.A., Zech, D. and Dorr, U, (1990) Cancer Pain Management According to WHO Analgesic Guidelines. Journal of Pain and Symptom Management, 5, 27-32. http://dx.doi.org/10.1016/S0885-3924(05)80006-5

[13] McGrath, P.A. (1996) Development of the World Health Organization Guidelines on Cancer Pain Relief and Palliative Care in Children. Journal of Pain and Symptom Management, 12, 87-92. http://dx.doi.org/10.1016/0885-3924(96)00099-1

[14] Cherny, N.I. (2000) The Management of Cancer Pain. CA: A Cancer Journal for Clinicians, 50, 70-116; Quiz 117-120. http://dx.doi.org/10.3322/canjclin.50.2.70

[15] Gordon, D.B., Dahl, J.L., Miaskowski, C., McCarberg, B., Todd, K.H., Paice, J.A., et al. (2005) American Pain Society Recommendations for Improving the Quality of Acute and Cancer Pain Management: American Pain Society Quality of Care Task Force. Archives of Internal Medicine, 165, 1574-1580. http://dx.doi.org/10.1001/archinte.165.14.1574

[16] Sapir, R., Catane, R., Kaufman, B., Isacson, R., Segal, A., Wein, S., Cherny, N.I., et al. (2000) Cancer Patient Expectations of and Communication with Oncologists and Oncology Nurses: The Experience of an Integrated Oncology and Palliative Care Service. Support Care Cancer, 8, 458-463. http://dx.doi.org/10.1007/s005200000163

[17] Induru, R.R. and Lagman, R.L. (2011) Managing Cancer Pain: Frequently Asked Questions. Cleveland Clinic Journal of Medicine, 78, 449-464. http://dx.doi.org/10.3949/ccjm.78a.10054

[18] Bernardi, M., Catania, G., Lambert, A., Tridello, G. and Luzzani, M. (2007) Knowledge and Attitudes about Cancer Pain Management: A National Survey of Italian Oncology Nurses. European Journal of Oncology Nursing, 11, 272279. http://dx.doi.org/10.1016/j.ejon.2006.09.003

[19] Yildirim, Y.K., Cicek, F. and Uyar, M. (2008) Knowledge and Attitudes of Turkish Oncology Nurses about Cancer Pain Management. Pain Management Nursing, 9, 17-25. http://dx.doi.org/10.1016/j.pmn.2007.09.002

[20] Shahnazi, H., Saryazdi, H., Sharifirad, G., Hasanzadeh, A., Charkazi, A., Moodi, M., et al. (2012) The Survey of Nurse's Knowledge and Attitude toward Cancer Pain Management: Application of Health Belief Model. Journal of Education and Health Promotion, 1, 15. http://dx.doi.org/10.4103/2277-9531.98573

[21] Fairbrother, G., Jastrzab, G., Kerr, S. and McInerney, M. (2003) Profiling the "Pain Aware” Nurse: Acute Care Nurses’ Attitudes and Knowledge Concerning Adult Pain Management. Australian Journal of Advanced Nursing, 21, 27-32.

[22] Soden, K., Ali, S., Alloway, L., Barclay, D., Perkins, P. and Barker, S. (2010) How Do Nurses Assess and Manage Breakthrough Pain in Specialist Palliative Care Inpatient Units? A Multicentre Study. Palliative Medicine, 24, 294298.

[23] Rustøen, T., Geerling, J.I., Pappa, T., Rundström, C., Weisse, I., Williams, S.C., Zavratnik, B., Kongsgaard, U.E. and Wengström, Y. (2013) A European Survey of Oncology Nurse Breakthrough Cancer Pain Practices. European Journal of Oncology Nursing, 17, 95-100. http://dx.doi.org/10.1016/j.ejon.2012.05.005

[24] González-Escalada, J.R., Camba, A., Casas, A., Gascón, P., Herruzo, I., Núñez-Olarte, J.M., et al. (2011) Código de buena práctica para el control del dolor oncológico [Good Practice Code for the Management of cancer Pain]. Revista de la Sociedad Española del Dolor, 18, 98-117. http://dx.doi.org/10.1016/s1134-248x(11)70011-8

[25] Owens, K. (2000) Effects of Nursing Education on Students' Pain Management Knowledge. Nurse Educator, 25, 33-37. http://dx.doi.org/10.1097/00006223-200001000-00008

[26] Rushton, P., Eggett, D., Carolyn, W. and Sutherland, S. (2003) Knowledge and Attitudes about Cancer Pain Management: A Comparison of Oncology and Nononcology Nurses. Oncology Nursing Forum, 30, 849-855. http://dx.doi.org/10.1188/03.ONF.849-855

[27] McMillan, S.C., Arn, P., Faan, M., Hagan, S.J. and Small, B.J. (2005) Training Pain Resource Nurses: Changes in Their Knowledge and Attitudes. Oncology Nursing Forum, 32, 890-902. http://dx.doi.org/10.1188/05.ONF.835-842

[28] King, R. (2004) Nurses' Perceptions of Their Pharmacology Educational Needs. Journal of Advanced Nursing, 45, 392-398. http://dx.doi.org/10.1046/j.1365-2648.2003.02922.x

[29] Plaisance, L. and Logan, C. (2006) Nursing Students’ Knowledge and Attitudes Regarding Pain. Pain Management Nursing, 7, 167-175. http://dx.doi.org/10.1016/j.pmn.2006.09.003

[30] El-Rahman, M.A., Al Kalaldeh, M.T. and Muhbes, F.J. (2013) Knowledge and Attitude towards Pain Management. A Comparison between Oncology and Non-Oncology Nurses in Jordan. International Journal of Advanced Nursing Studies, 2, 95-100.

[31] Fothergill-Bourbonnais, F. and Wilson-Barnett, B. (1992) A Comparative Study of Intensive Therapy Unit and Hospice Nurses’ Knowledge on Pain Management. Journal of Advanced Nursing, 17, 362-372.

http://dx.doi.org/10.1111/j.1365-2648.1992.tb01915.x 